\section{Child with Multiple Anomalies and a Group B(4-5) Long arm Deletion (Bq-)}

Though some six chromosome deletion syndromes have so far been delineated, with the exception of the 'cri-du-chat' syndrome ( $5 \mathrm{p}-)$, the total number of subjects described is small. Other instances of deletions have in almost all cases remained unique observations. We wish to report a new instance of a long arm deletion of a $\mathrm{B}(4-5)$ chromosome.

\section{Case Report}

The mother (aged 23) and father (aged 30) of the propositus were normal, healthy, and unrelated, and there was no family history of mental deficiency or congenital abnormality. This was the first pregnancy and it was uneventful apart from contact with rubella at 10 weeks. As the mother was unsure of having contracted the disease previously she was given $\gamma$-globulin intramuscularly 5 days later. The propositus, a male, birthweight $3030 \mathrm{~g}$ was delivered at term by forceps after a prolonged second stage, and was covered in meconium. He had an oddly shaped skull, pitting oedema of hands and feet, and he became limp and cyanosed during the first 8 hours of life. A soft systolic murmur was noted over the left second interspace while there was radiological evidence of right upper lobe collapse and small bilateral pneumothoraces. He was treated with oxygen, digitalis, and antibiotics with slight improvement. At the age of 10 days he was transferred to a specialist unit.

At that time he appeared an ill baby, coughing, and breathless. His weight was $3060 \mathrm{~g}$ and length $49 \cdot 5 \mathrm{~cm}$. The skull was abnormal with brachycephaly, a very large and sunken anterior fontanelle which was tilted anteriorly, a widely open metopic suture, prominent frontal bones, parietal bones contiguous across the sagittal suture, and a large posterior fontanelle. His head circumference was $35.5 \mathrm{~cm}$. The eyes were deeply set and small, with an iris width of $0.95 \mathrm{~cm}$ and an interpupillary distance of $4.2 \mathrm{~cm}$. No cataracts were seen but the fundi showed traces of black retinal pigment. The mandible was small. The ears were slightly low set and angulated and the upper half of the pinna showed a simple sculpting. The arms appeared short with ulnar angulation at the wrists and both little fingers were grossly incurved. Ulnar loops were present on both thumbs while all other digits had whorls. The left palm had a single axial triradius (atd angle $=47^{\circ}$ ) while two axial triradii were present on the right (atd angle $=98^{\circ}$ ). Loop patterns were found on both hypothenar eminences. A transverse palmar crease was present on the left, and the left little finger showed a single flexion crease. The femora appeared short. The right testis was undescended.

A systolic murmur was heard intermittently over the left 2nd to 4th interspace, but there were no signs of heart failure and the femoral pulses were normal. Medium rales were present over all areas of the chest and respirations were $96 /$ minute. He responded to bright light by blinking; neither fixation nor following were observed. He showed no definite response to sound and he was very hypotonic, with a weak grasp and absent Moro reflex.

Investigations. Serial $x$-rays revealed spontaneous regression of the pneumothoraces and the heart contour was normal. The 6th dorsal vertebra was cleft coronally. The vault of the skull and facial bone development were abnormal. The humeri and femora were normal. An electrocardiogram showed $3.5 \mathrm{~mm} P$ pulmonale and biventricular hypertrophy. Rubella haemagglutination inhibition titre was initially $1 / 128$ and fell to $1 / 32$ at 2 months, and the mother's titre was $1 / 64$, thus suggesting that infection had not occurred during pregnancy. The toxoplasma dye test titre was $1 / 32$ and the cytomegalovirus titre was less than $1 / 8$. Viruses were not isolated from stool, urine, or throat swabs. $\mathrm{Hb}$ was $20 \mathrm{~g} / 100 \mathrm{ml}$ and WBC $24,000 / \mathrm{mm}^{3}$ with $78 \%$ neutrophils.

Progress. He recovered from his respiratory difficulties during his 4 th week but remained a hypotonic, unresponsive baby, and was transferred to a long-stay ward. He retained his abnormal appearance and, though his anterior fontanelle pressure remained low, prominent veins appeared over his scalp. On examination at 9 months (Fig. 1) he was noted to adopt a persistently supine position with arms flexed and legs extended. He showed complete head lag when his shoulders were raised from the cot. His visual and auditory responses had altered little since the neonatal period and he had never been observed to smile. No heart murmur was heard and his chest sounded clear though he was snuffly. The right testis was at the upper end of the inguinal canal. His measurements at this time were head circumference $43 \mathrm{~cm}$, length $61 \mathrm{~cm}$, and weight $4400 \mathrm{~g}$. Within 48 hours of this examination he died of an acute respiratory illness.

Necropsy. The cut surface of the lungs showed oozing pus and signs of chronic pulmonary infection. 


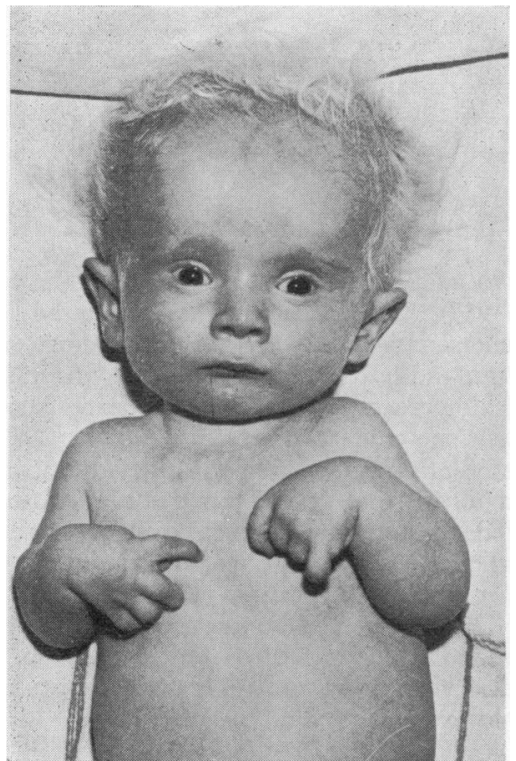

FIG. 1.-Patient aged 9 months showing tall forehead, large cranial vault, low set ears, and characteristic posture of arms and hands.

The heart was dilated and flabby but there were no congenital malformations. The brain weighed $510 \mathrm{~g}$ and showed diffuse polymicrogyria and agenesis of the corpus callosum. There was a deficiency of cerebro- spinal fluid. The right kidney was twice the normal size while the left was normal.

\section{Cytogenetic Studies}

Analysis based on cultured peripheral blood revealed 46 chromosomes with XY sex chromosomes. Only 3 normal chromosomes were present in group B (4-5), and there was apparently an additional chromosome in group C (X 6-12). All other groups were normal, there being no evidence of a balanced translocation involving the B group. The karyotype interpretation is therefore 46, XY, Bq- (Fig. 2). Measurements indicate that between 25 and $30 \%$ has been lost.

Tritiated thymidine (conc. $0.5 \mathrm{Ci} / \mathrm{ml}$ ) was added to other blood cultures for autoradiographic studies. Of the 14 cells selected for analysis, 6 were informative and showed 2 group B chromosomes with late labelling and one with early labelling. This suggests that the deleted chromosome is No. 5 .

Peripheral blood cultures from both parents produced normal karyotypes.

\section{Comment}

An extensive search of the literature has revealed only 4 patients with abnormalities involving a possible deficiency of the long arms of a $B(4-5)$ chromosome, and 3 of these had ring chromosomes. In the first reported by Steele et al. (1966), the patient had clinical features of the cri-du-chat syndrome, and the deletion probably involved mostly the short arm of No. 5. The other two,

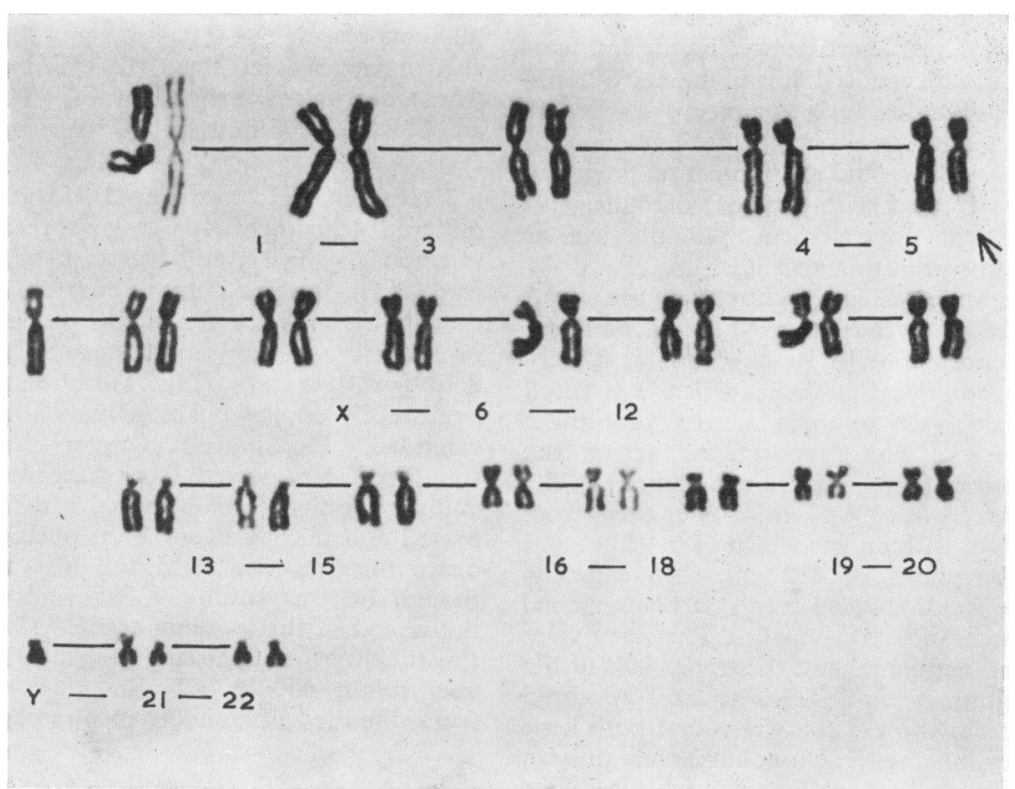

FIG. 2.-Karyotype of cultured lymphocyte showing a group $B$ long arm deletion, 46, $X Y, B q-$ (arrow). 
reported by Carter, Baker, and Hayman (1969) and Faed, Stewart, and Keay (1969) had many features in common with the $4 \mathrm{p}$ - syndrome but the latter, with 45, XY, B-/46, XY, Br mosaicism had bilateral absence of radius and thumb in addition. The only patient with apparently a simple deletion of the long arm of a B group chromosome (Ockey et al., 1967) also had an absent left radius. Only a single digit was present on the left hand, and cardiac anomalies (atrial and ventricular septal defects) were observed at necropsy. The deleted chromsome was proven to be No. 4 on the basis of an autoradiographic analysis.

Our patient, therefore, differs in many respects from all these cases and seems to represent a new clinical entity.

\section{Summary}

A child with multiple anomalies and a $46, \mathrm{XY}$, B5q- karyotype is described.

We would like to thank Dr. I. M. Anderson for permission to publish his case and Dr. G. W. Fisher, Medical Superintendent, Cell Barnes Hospital, for necropsy details.

\section{REFERENCES}

Carter, R., Baker, E., and Hayman, D. (1969). Congenital malformations associated with a ring 4 chromosome. fournal of Medical Genetics, 6, 224.

Faed, M., Stewart, A., and Keay, A. J. (1969). Chromosome abnormalities in two cases with bilateral radial element defects. fournal of Medical Genetics, 6, 342.

Ockey, C. H., Feldman, G. V., Macaulay, M. E., and Delaney, M. J. (1967). A large deletion of the long arms of chromosome No. 4 in a child with limb abnormalities. Archives of Disease in Childhood, 42, 428.

Steele, M. W., Breg, W. R., Eidelman, A. I., Lion, D. T., and Terzakis, T. A. (1966). A B-group ring chromosome with mosaicism in a newborn with cri-du-chat syndrome. Cytogenetics, 5, 419.

\section{Addendum}

The mother has recently given birth to a normal boy following the prediction of a normal male karyotype $(46, \mathrm{XY})$ by amniotic fluid cell culture at 15 weeks' gestation.

R. H. LINDENBAUM and L. J. BUTLER

Westminster Hospital, London SW1, and Queen Elizabeth Hospital for Children, London E2.

\section{Congenital Left Coronary Artery Fistula Draining into Right Atrium \\ An Uncommon Cause of Continuous Murmur in Childhood}

Congenital coronary arterial fistula draining into a cardiac chamber or great vessels is a rare cause of a continuous murmur in children (Moss and Adams,
1968). Surgical correction of these lesions has a very low operative mortality and is curative (Taber, Gale, and Lam, 1967; Kahn, Strang, and Wilson, 1968). This paper reports a case of congenital left coronary arterial fistula draining into the right atrium in a child and discusses the salient features of diagnosis and treatment.

\section{Case Report}

This 5-year-old, asymptomatic, Chinese boy was referred to the paediatric service of Grace-New Haven Hospital, for evaluation of a heart murmur that was heard at $3 \frac{1}{2}$ years of age during a routine physical checkup.

Physical examination. He had normal vital signs and a blood pressure of $90 / 60 \mathrm{~mm} \mathrm{Hg}$. There was no cyanosis or clubbing. Pertinent cardiac findings included a faint systolic thrill and a continuous murmur over the fourth right intercostal space, parasternally. The second heart sound was physiologically split with prominence of the pulmonary component.

Investigations. Chest $x$-ray showed right ventricular hypertrophy, increase in pulmonary vasculature, and a prominent aorta. Electrocardiogram showed right axis deviation.

Right heart catheterization. A significant step-up in oxygen saturation was noted in the right atrium indicating a left-to-right shunt at this level such that the pulmonary blood flow was 1.5 times the systemic flow. The pressures in the right heart and pulmonary artery were normal.

Phonocardiography. External phonocardiography showed a continuous murmur at the fourth right intercostal space, parasternally. Intracardiac phonocardiographic studies recorded a continuous murmur in the right atrium which disappeared on withdrawing the catheter to inferior vena cava.

Angiocardiography. Injection of the contrast material in the left atrium did not show any evidence of a left-to-right shunt at atrial or ventricular level. The ascending aorta was slightly dilated. The right coronary artery was normal. A branch of the left coronary artery was dilated and followed an abnormal course along the posterior border of the right atrium. The late phase of the angiocardiogram showed delayed emptying of this branch into the right atrium through a fistulous opening (Figure).

Surgery. The operation was done by Dr. H. C. Stansel, using cardiopulmonary bypass. Cardiac exploration showed a large right atrium with a prominent thrill on its surface. A markedly dilated tortuous branch of the left coronary artery was seen with fistulous opening $(1.3$ to $1.5 \mathrm{~cm})$ into the posterior aspect of right atrium, at the level of foramen ovale. The abnormal coronary artery was ligated first at the fistula 